\title{
How Rewards in Exercise Games Affect Intrinsic Motivation
}

\author{
Ying Wang ${ }^{1}$ Yue-sheng Li Lin-lin Chen \\ Universal Design Institute, School of Art and Design, Zhejiang Sci-tech University
}

\begin{abstract}
The design of reasonable reward mechanisms in exercise games aims to increase people's intrinsic motivation to participate in the games for training so that they can persist for a long time and achieve exercise results. This study developed two game modes based on a prototype of an exercises game for a Kinect interactive device. Two reward mechanisms, in-game rewards and extrinsic rewards, were included in one of the game modes respectively. Twelve older subjects and twelve younger subjects were recruited to test the game. They rated the game using an intrinsic motivation scale. The results showed that young people who played the ingame reward mode scored higher on the motivation scale. In contrast to young people, older people had higher motivation levels when playing the extrinsic reward mode. The results highlight the variability in the effects of in-game and extrinsic rewards on intrinsic motivation across age groups and can help people better design reward mechanisms when developing exercise games.
\end{abstract}

Keywords. Exercise games, rewards mechanisms, intrinsic motivation

\section{Introduction}

As a type of serious game, exercise games have been shown to significantly improve the effectiveness of people's rehabilitation and exercise. Rehabilitation and exercise is lengthy process for participants. If they lack sufficient willingness to engage in play, they may stop halfway. Therefore, it is important to determine what methods should be used to stimulate and encourage the active participation of older people in the rehabilitation and exercise process. There are many studies on exercise-based serious games nowadays. However, there is relatively little discussion on the motivational aspects of such games.

Rewards are often applied to serious games that promote learning, habit formation, or behavior change. However, rewards may inhibit participants' intrinsic motivation and keep them from persisting over time ${ }^{[1]}$. Gamification is the use of game design elements in a non-game environment ${ }^{[2]}$. Gamification attempts to use the motivation of playing games to promote engagement, persistence, and achievement ${ }^{[3]}$. Anant Vaibhav et al. increased user enrollment and user engagement in MOOCs educational courses by making the learning tools gamification while making the learning process a unique and fun experience ${ }^{[4]}$. A study by Jonna Koivisto et al. on gamification interventions for older

${ }^{1}$ Ying Wang, Universal Design Institute, School of Art and Design, Zhejiang Sci-tech University; Email: winered@zstu.edu.cn. 
adults suggests that users may benefit from gamification and game-based interventions, especially in the health domain ${ }^{[5]}$.

Rewards are usually divided into intrinsic and extrinsic rewards based on how individuals perceive and receive them ${ }^{[6]}$. The intrinsic rewards referred to in this paper are the in-game rewards. Hallford describes four different types of game rewards: Reward of Access, Reward of Facility, Reward of Sustenance, and Reward of Glory ${ }^{[7]}$. The reward of Access allows players to continually unlock new scenarios, resources, and more. The reward of Facility is often associated with game systems, such as allowing players to upgrade and gain more powerful abilities. The reward of Sustenance extends the amount of time a player can play by giving them extra lives or health. Rewards of Sustenance are given so that the player can maintain their avatar's status quo and keep the possessions that they have gained in the game so far ${ }^{[7]}$. The reward of Glory does not affect the game's progress. However, it will give players a sense of purpose and accomplishment - for example, points, gold, achievement walls, level medals. Extrinsic rewards are related to extrinsic motivation, which refers to pushing players to do or not do something through external force, such as a reward or punishment ${ }^{[8]}$. Extrinsic motivation depends on how well the activity aligns with our goals ${ }^{[9]}$. In contrast, intrinsic motivation is related to an individual's desires, interests, aspirations, and tastes. The activity itself can be a strong motivator for an individual to go and engage in $\mathrm{it}^{[10]}$. Existing research is inconclusive about the relationship between extrinsic and intrinsic motivation, and there is no consensus on the effect of in-game versus extrinsic rewards on intrinsic motivation. In this paper, two different game modes, in-game and extrinsic rewards, were used to evaluate the effects of in-game and extrinsic rewards on intrinsic motivation in two different populations, young and old, based on a serious game of exercise.

\section{Methods}

The study used a 2-by-2 between-group experimental design, with game mode as the independent variable. Two game modes were compared, and the dependent measures were: (1) Intrinsic Motivation Inventory; (2) duration of gameplay; (3) number of rounds played.

\subsection{Participants}

Twelve older adults between 60 and 78 years of age and twelve young adults between 21 and 24 years of age participated in an experimental study(Table 1). Participants were excluded if they were unable to follow instructions given by the researchers or if they failed to achieve a score of 26 on the Montreal Cognitive Assessment. The young participants were all from Zhejiang Sci-Tech University. Older participants were from communities around the university.

\subsection{Apparatus}

The experimental setup consists of a Kinect sensor and a PC. The game is run through the Unity game engine on the PC. Real-time data of image, depth, and skeleton position is obtained from the Kinect sensor and processed by the PC software. The player only 
needs to stand about one meter away from the Kinect sensor to make their hand movements can be captured. A few seconds after the game starts, the player's skeleton will be tracked, and the player can play the game through pose recognition.

Table 1. Participant characteristics

\begin{tabular}{lllll}
\hline & & Mean & SD & Range \\
\hline \multirow{3}{*}{ Old adults } & Female $(\mathrm{N}=7)$ & 68.43 & 5.62 & $60-78$ \\
& Male $(\mathrm{N}=5)$ & 67.2 & 3.83 & $62-72$ \\
& Total $(\mathrm{N}=12)$ & 67.9 & 4.80 & $60-78$ \\
\cline { 2 - 5 } Young adults & Female $(\mathrm{N}=6)$ & 21.67 & 0.52 & $21-22$ \\
& Male $(\mathrm{N}=6)$ & 23.17 & 0.75 & $22-24$ \\
& Total $(\mathrm{N}=12)$ & 22.42 & 1.00 & $21-24$ \\
\hline
\end{tabular}

\subsection{Procedure}

All participants played an upper limb training exergame. It is a parkour game. The goal of the game is to control the characters in the game, running forward to avoid obstacles. Players wave their arms to the left and right, and the characters in the game will move in the same direction. During the game, players only need to wave their arms without moving their bodies.

The game is divided into two modes. In the first game mode, gold coins and special props such as "magnet" and "acceleration" will appear randomly on the road. The number of gold coins collected by players will be displayed in the upper left corner of the game screen in real-time. The props and the remaining time of the props obtained will be displayed on the right side of the screen(Fig.1). In the second game mode, there are no gold coins, and no special props, the character in the game is just running on a road with obstacles(Fig.2).

Finally, participants completed a series of experimental trials. Twelve older adults and 12 young people were respectively divided into two groups of six people each. One group of participants played the first mode of the game. The participants only knew that it was a somatic interactive parkour game and did not know it was an exergame. Another group of participants played the second mode of the game. The participants were told that it was an exergame to exercise the upper limbs. If the participants could complete the exercise seriously, they would be rewarded in kind.

All participants completed an informed consent form before the formal experiment and completed the Montreal Cognitive Assessment with a score of 26 or higher. In the experiment, participants were allowed to try out the game first to familiarize themselves with the operation of the somatic interaction. A formal game was followed then. Participants were told to play the game as much as possible but could opt-out at any time. The experiment would measure the duration of gameplay and the number of rounds they played without their knowledge. After the game, participants filled out the IMI. They were asked four questions related to the game, which were: what participants liked (Q1) or disliked (Q2) about the game, what prevented them from playing (Q3), and if they had additional remarks $(\mathrm{Q} 4)^{[11]}$. 


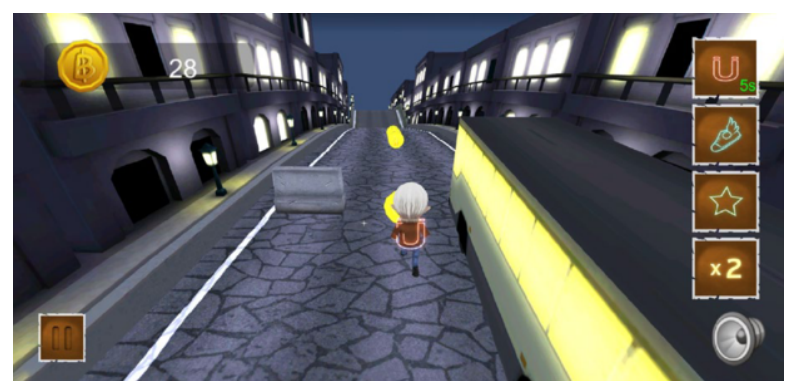

Figure 1. In-game reward model.

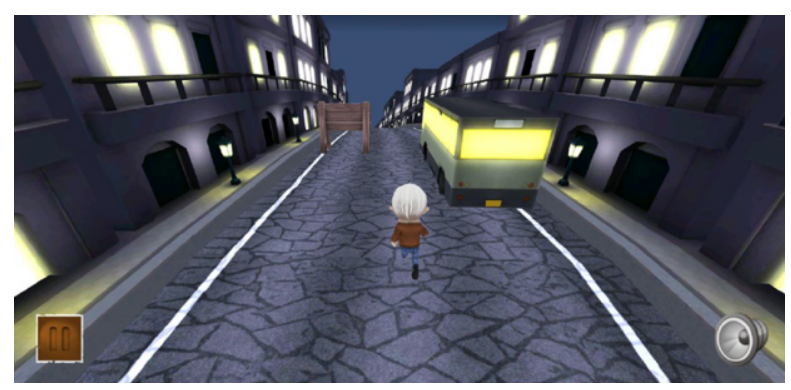

Figure 2. Extrinsic reward model.

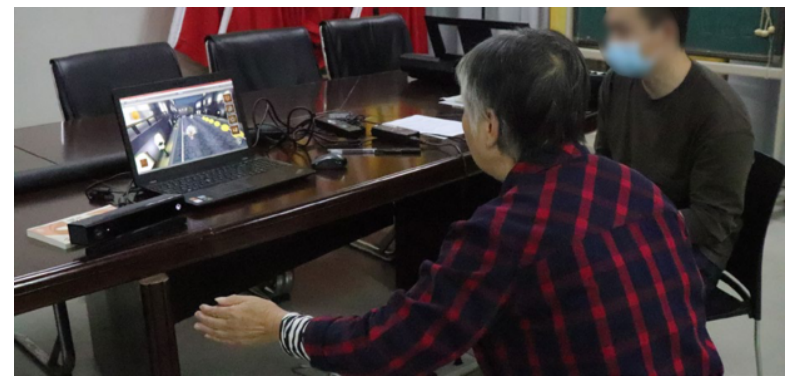

Figure 3. Participation of older adults in experiments.

\section{Results}

On average, older adults who played games that included extrinsic rewards showed the highest interest and enjoyment. They had a better perception of the game and put more effort into playing the game. Moreover, they also gave the highest scores to the value of the game(Fig.4). Two-way ANOVA's were conducted to evaluate the effect of rewards type and age group on the seven scalar outcome measures (Table 2). No significant reward main effects or significant interactions between reward and age group were found. However, the age group had a significant main effect on the two scalar measures: Interest/Enjoyment and Value/Usefulness. The older group scored higher than the younger group in both categories. 


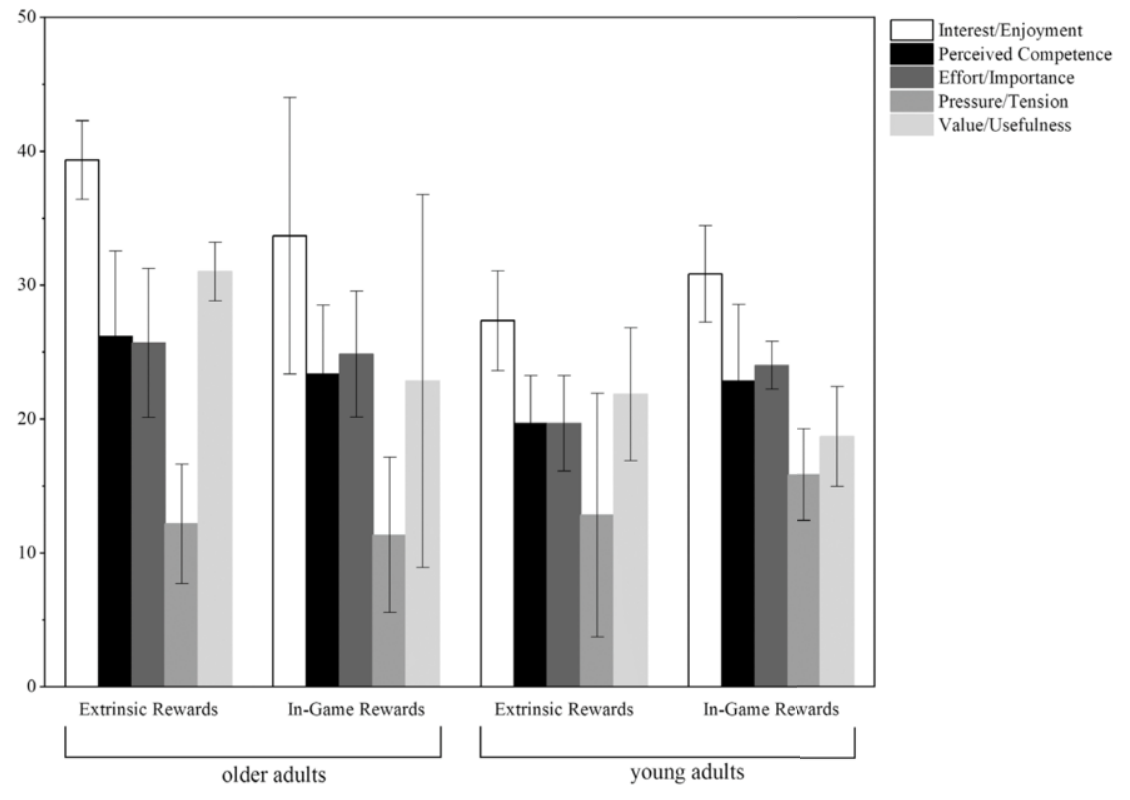

Figure 4. Mean and standard deviation for interest/enjoyment, perceived competence, effort/importance, pressure/tension, value/usefulness.

Table 2. Results of two-way ANOVA to evaluate the effect of interest/enjoyment, perceived competence, effort/importance, pressure/tension, value/usefulness, duration of gameplay and rounds

\begin{tabular}{|c|c|c|c|c|c|c|c|c|}
\hline Dimension & & $\begin{array}{l}\text { Interest/ } \\
\text { Enjoyment }\end{array}$ & $\begin{array}{l}\text { Perceived } \\
\text { Competence }\end{array}$ & $\begin{array}{l}\text { Effort/ } \\
\text { Importance }\end{array}$ & $\begin{array}{l}\text { Pressure/ } \\
\text { Tension }\end{array}$ & $\begin{array}{l}\text { Value/ } \\
\text { Usefulness }\end{array}$ & $\begin{array}{l}\text { Duration } \\
\text { of } \\
\text { Gameplay }\end{array}$ & Rounds \\
\hline \multirow{3}{*}{$\begin{array}{l}\text { Rewards } \\
\text { (extrinsic/ } \\
\text { in-game) } \\
\text { df: } 1,20\end{array}$} & $\mathrm{~F}$ & .198 & .000 & 1.064 & .191 & 3.252 & .120 & .176 \\
\hline & $\mathrm{P}$ & .661 & 1.000 & .315 & .667 & .086 & .733 & .679 \\
\hline & $\eta^{2}$ & .010 & .000 & .050 & .009 & .140 & .006 & .009 \\
\hline \multirow{3}{*}{$\begin{array}{l}\text { Group } \\
\text { (old adults/ } \\
\text { young adults) } \\
\text { df: } 1,20\end{array}$} & $\mathrm{~F}$ & 9.286 & 1.870 & 4.055 & 1.085 & 4.501 & 2.488 & 3.152 \\
\hline & $\mathrm{P}$ & $.006^{*}$ & .187 & .058 & .310 & $.047^{*}$ & .130 & .091 \\
\hline & $\eta^{2}$ & .317 & .086 & .169 & .051 & .184 & .111 & .136 \\
\hline \multirow{3}{*}{$\begin{array}{l}\text { Rewards } \times \\
\text { group } \\
\text { df: } 1,20\end{array}$} & $\mathrm{~F}$ & 3.546 & 1.351 & 2.318 & .597 & .633 & .230 & 1.751 \\
\hline & $\mathrm{P}$ & .074 & .259 & .144 & .449 & .436 & .637 & .201 \\
\hline & $\eta^{2}$ & .151 & .063 & .104 & .029 & .031 & .011 & .081 \\
\hline
\end{tabular}

*Statistically significant at the $\mathrm{P}<.05$ level 
Independent-samples t-tests were conducted separately for the young and old groups. In the young group, the reward category significantly affected only one scalar measure: Effort/Importance(Table 3). Younger people who played the in-game reward model were more engaged and put more effort into playing the game. Three of those who answered the exit question explicitly expressed concern about gold, points, props, levels, and scenarios and wanted to perform better in the game. Among older adults, reward categories did not have a significant effect on the seven scalar measures(Table 4).

Table 3. Independent-samples t-test for the young group

\begin{tabular}{lllll}
\hline Dimension & Extrinsic(n=6) & In-Game(n=6) & \multicolumn{2}{c}{ Independent-samples t-test } \\
\hline & Mean(S.D.) & Mean(S.D.) & t value & Sig. (two-sided) \\
\hline Interest/Enjoyment & $27.33(3.72)$ & $30.83(3.60)$ & -1.655 & .129 \\
Perceived Competence & $20.00(6.51)$ & $22.83(5.74)$ & -.799 & .443 \\
Effort/Importance & $19.67(3.56)$ & $24.00(1.79)$ & -2.665 & $.024^{*}$ \\
Pressure/Tension & $12.83(9.09)$ & $15.83(3.43)$ & -.757 & .467 \\
Value/Usefulness & $21.83(4.96)$ & $18.67(3.72)$ & 1.251 & .239 \\
Duration of Gameplay & $492.17(393.57)$ & $476.83(380.38)$ & .069 & .947 \\
Rounds & $11.83(11.69)$ & $7.33(3.98)$ & .893 & .393 \\
\hline
\end{tabular}

*Statistically significant at the $\mathrm{P}<.05$ level

Table 4. Independent-samples t-test for the old group

\begin{tabular}{lllll}
\hline Dimension & Extrinsic(n=6) & In-Game(n=6) & \multicolumn{2}{l}{ Independent-samples t-test } \\
\hline & Mean(S.D.) & Mean(S.D.) & t value & Sig. (two-sided) \\
\hline Interest/Enjoyment & $39.33(2.94)$ & $33.67(10.33)$ & 1.292 & .225 \\
Perceived Competence & $26.17(6.37)$ & $23.33(5.16)$ & .846 & .417 \\
Effort/Importance & $25.67(5.57)$ & $24.83(4.71)$ & .280 & .785 \\
Pressure/Tension & $12.17(4.45)$ & $11.33(5.79)$ & .280 & .785 \\
Value/Usefulness & $31.00(2.19)$ & $22.83(13.92)$ & 1.420 & .212 \\
Duration of Gameplay & $255.50(70.89)$ & $350.50(114.58)$ & -1.727 & .115 \\
Rounds & $3.83(1.47)$ & $6.17(2.32)$ & -2.082 & .064 \\
\hline
\end{tabular}

In the last step of the study procedure, the participants finished the exit questionnaire. In the in-game reward group, 6/12 participants positively commented on in-game rewards such as coins, points, and props. In comparison, 9/12 participants in the extrinsic reward group said that the game became boring after a while. They think levels should be set in the game, difficulty should be increased, or new game scenes added. In addition, $7 / 12$ participants in the extrinsic reward group thought playing the game could get an 
exercise effect. All 6 participants in the elderly group agreed with this. Two older adults in the in-game reward group also expressed the same opinion. The young people in the extrinsic reward group (3/6), on the other hand, focused more on novel interaction methods and less on the exercise effect of the game.

\section{Discussion and conclusions}

A somatic interactive parkour game was divided into two game modes: in-game rewards and extrinsic rewards. Twelve young people and twelve older people were randomly selected to participate in the game experiment. Statistical analysis of the IMI and objective measures revealed that reward did not significantly affect the five dimensions of intrinsic motivation, nor did it significantly affect the duration of gameplay or the number of rounds played. However, the age group had a significant effect on interest and value. The higher ratings given by older adults on both interest and value dimensions may be because older users are more likely to play for the rewards. In contrast, younger users are more likely to just come to pass the time ${ }^{[12]}$. Some studies have shown that older adults are less sensitive to immediate rewards in the decision-making process ${ }^{[13]}$ and have a greater willingness to wait for delayed rewards ${ }^{[14,15]}$. This statement is also consistent with the post hoc statements of older adults. Older adults in the extrinsic reward game group rated and anticipated the availability of exercise positively as a reward, which was not the case among the younger adults in the same group.

Although we emphasize that the effect of the two reward modes on intrinsic motivation in the present study was not statistically significant, there is still something meaningful to be found. In the young adult group, those who played the in-game reward mode gave higher scores on all four scalar measures of interest enjoyment, perceived competence, effort, and stress. In comparison, those who played the extrinsic reward mode gave higher scores only on the value and usefulness measures. The theory that extrinsic rewards undermine intrinsic motivation - a phenomenon called the undermining effect $^{[16]}$ - is a plausible explanation for these results. Surprisingly, in the older group, those who played the extrinsic reward mode scored higher on all five items, possibly because older people developed a subjective preference after knowing that it was an exercise game. However, the objective metric still showed that they played for a shorter time and with fewer rounds than those who played the in-game reward mode.

This study has several limitations. Firstly, the study examined the effect of rewards on players' intrinsic motivation in exercise games at only one point in time. Exercise games often require long periods of regular persistence. Eysenbach argues that in healthrelated applications, most users give up before completing the expected course of use, which he refers to as "the law of attrition"[17]. Therefore, this study cannot conclude the long-term effects of rewards on intrinsic motivation, which are essential for studying the effects of rewards on intrinsic motivation. Secondly, the participants in this study were all from the same region. They had similar cultural backgrounds, so cultural differences could not be considered in the results.

Despite its limitations, the present study examines the effects of two types of rewards on players' intrinsic motivation in exercise games and has implications for the design of rewards in future exercise games. A possible direction for future research is to evaluate the long-term effects of extrinsic versus in-game rewards on players' intrinsic motivation. The results of the present study suggest that investigating the effects of not only the type 
of rewards but also how their effects on intrinsic motivation change over time is a feasible avenue for future research.

\section{Funding}

This research was supported by the National College Students Innovation and Entrepreneurship Training Program (No. 202010338065X).

\section{References}

[1] Lewis Z H, Swartz M C, Lyons E J. What's the point?: a review of reward systems implemented in gamification interventions [J]. Games for health journal, 2016, 5(2): 93-9.

[2] Deterding S, Dixon D, Khaled R, et al. From game design elements to gamefulness: defining "gamification"; Proceedings of the 15th international academic MindTrek conference: Envisioning future media environments, F, 2011 [C].

[3] Richter G, Raban D R, Rafaeli S. Studying gamification: the effect of rewards and incentives on motivation [M]. Gamification in education and business. Springer. 2015: 21-46.

[4] Vaibhav A, Gupta P. Gamification of MOOCs for increasing user engagement; proceedings of the 2014 IEEE International Conference on MOOC, Innovation and Technology in Education (MITE), F, 2014 [C]. IEEE.

[5] Koivisto J, Malik A. Gamification for older adults: a systematic literature review [J]. The Gerontologist, 2021, 61(7): e360-e72.

[6] Cameron J, Pierce W D. Reinforcement, reward, and intrinsic motivation: A meta-analysis [J]. Review of Educational research, 1994, 64(3): 363-423.

[7] Hallford N, Hallford J. Swords and circuitry: A designer's guide to computer role-playing games [M]. Premier Press, 2001.

[8] Cruz C, Hanus M D, Fox J. The need to achieve: Players' perceptions and uses of extrinsic meta-game reward systems for video game consoles [J]. Computers in Human Behavior, 2017, 71: 516-24.

[9] Birk M V, Mandryk R L, Atkins C. The motivational push of games: The interplay of intrinsic motivation and external rewards in games for training; Proceedings of the 2016 Annual Symposium on ComputerHuman Interaction in Play, F, 2016 [C].

[10] Cota T T, Ishitani L, Vieira Jr N. Mobile game design for the elderly: A study with focus on the motivation to play [J]. Computers in Human Behavior, 2015, 51: 96-105.

[11] Alexandrovsky D, Friehs M A, Grittner J, et al. Serious Snacking: A Survival Analysis of how Snacking Mechanics Affect Attrition in a Mobile Serious Game; Proceedings of the $2021 \mathrm{CHI}$ Conference on Human Factors in Computing Systems, F, 2021 [C].

[12] Wohn D Y, Lee Y-H. Players of facebook games and how they play [J]. Entertainment Computing, 2013, 4(3): 171-8.

[13] Eppinger B, Nystrom L E, Cohen J D. Reduced sensitivity to immediate reward during decision-making in older than younger adults [J]. PloS one, 2012, 7(5): e36953.

[14] Green L, Myerson J, Ostaszewski P. Discounting of delayed rewards across the life span: age differences in individual discounting functions [J]. Behavioural Processes, 1999, 46(1): 89-96.

[15] Reimers S, Maylor E A, Stewart N, et al. Associations between a one-shot delay discounting measure and age, income, education and real-world impulsive behavior [J]. Personality and Individual Differences, 2009, 47(8): 973-8.

[16] Lepper M R, Greene D, Nisbett R E. Undermining children's intrinsic interest with extrinsic reward: A test of the "over-justification" hypothesis [J]. Journal of Personality and social Psychology, 1973, 28(1): 129.

[17] Eysenbach G. The law of attrition [J]. Journal of medical Internet research, 2005, 7(1): e402. 